\title{
Síndrome de Kinsbourne manifestando-se com quadro de encefalite pós-viral
}

\author{
Kinsbourne syndrome manifesting with signs of post-viral encephalitis
}

Olberes Vitor B. Andrade ${ }^{1}$, Eduardo Juan Troster ${ }^{2}$, Saul Cype/ ${ }^{3}$

\section{RESUMO}

Objetivo: Descrever um caso de síndrome de Kinsbourne manifestando-se com quadro de encefalite pós-viral e rever a da literatura.

Descrição do caso: Criança do sexo feminino, dois anos e seis meses, encaminhada de outro serviço com história de ataxia, irritabilidade e dificuldades articulatórias na fala após episódio prodrômico de febre, lesões de pele e mucosa. Com hipótese de encefalite pós-viral, a avaliação clínica evidenciou quadro de síndrome opsoclônus-mioclonia-ataxia ou síndrome de Kinsbourne. Foi afastada a associação de neuroblastoma oculto e iniciada terapêutica com corticosteroide. Durante internação e acompanhamento ambulatorial, houve regressão progressiva e normalização do quadro clínico e neurológico inicial.

Comentários: Apesar de se tratar de uma doença rara, o diagnóstico de síndrome de Kinsbourne deve ser reconhecido pelos pediatras e intensivistas, com objetivo de instituir tratamento específico precoce, embora com resultados variáveis, sendo fundamental a exclusão de neuroblastoma oculto.

Palavras-chave: ataxia; mucolipidoses; transtornos da motilidade ocular; dexametasona

\section{ABSTRACT}

Objective: To describe a case of Kinsbourne syndrome manifesting with signs of post-viral encephalitis, and to review the literature.

Case description: Female child, aged two years and six months. She was referred from another hospital with a history of ataxia, irritability, and dysphasia after a prodromal episode of fever, skin and mucosa lesions. Referred with suspected post-viral encephalitis, the child was diagnosed with the opsoclonus-myoclonus-ataxia syndrome (Kinsbourne syndrome). The association of occult neuroblastoma was dismissed and therapy with corticosteroids was initiated. During hospitalization and outpatient treatment, there was a progressive regression and normalization of the clinical and neurological original condition.

Comments: Albeit a rare disease, the diagnosis of Kinsbourne syndrome should be recognized by pediatricians and intensivists in order to start an early specific treatment, being important to exclude occult neuroblastomas. The results of the treatment are variable.

Key-words: ataxia; mucolipidoses; ocular motility disorders; dexamethasone.
Instituição: Centro de Terapia Intensiva Pediátrica do Hospital Israelita Albert Einstein (HIAE), São Paulo, SP, Brasil

'Doutor pela Faculdade de Ciências Médicas da Santa Casa de São Paulo; Médico do Centro de Terapia Intensiva Pediátrica do HIAE, São Paulo, SP, Brasil

2Livre-Docente pelo Departamento de Pediatria da Faculdade de Medicina da Universidade de São Paulo (USP); Coordenador Médico do Centro de Terapia Intensiva Pediátrica do HIAE, São Paulo, SP, Brasil

${ }^{3}$ Livre-Docente pelo Departamento de Neurologia da Faculdade de Medicina da USP; Neurologista Infantil do HIAE, São Paulo, SP, Brasil
Endereço para correspondência:

Olberes Vitor B. Andrade

Rua Desembargador Joaquim Barbosa de Almeida, 95 - Alto de Pinheiros CEP 05463-010 - São Paulo/SP

E-mail: olberes@uol.com.br

Conflito de interesse: nada a declarar

Recebido em: 14/7/2010

Aprovado em: 14/12/2010 


\section{Introdução}

A síndrome de Kinsbourne (SK) é uma enfermidade neurológica rara, frequentemente subdiagnosticada, que apresenta um amplo espectro de manifestações neurológicas de caráter agudo ou crônico ${ }^{(1)}$. Clinicamente, evidencia-se a presença de uma tríade composta de movimentos oculares conjugados rápidos, anárquicos e multidirecionais (opsoclonos), abalos musculares erráticos (polimioclonias) e ataxia cerebelar axial e apendicular ${ }^{(1,2)}$. Outras denominações também utilizadas são síndrome opsomioclônica, ataxia oposoclonus-mioclônica, síndrome opsóclono-mioclonia-ataxia, síndrome opsocloniamioclonia-ataxia, síndrome opsoclonus-mioclonus, síndrome opsoclonus-mioclonus-ataxia, encefalopatia opsoclônica, encefalopatia mioclônica da infância e síndrome da "dança dos

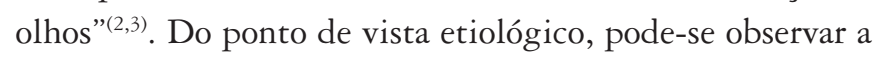
identificação de casos idiopáticos, a associação com infecções virais, doenças autoimunes e, em $50 \%$ dos casos, neuroblastoma oculto ${ }^{(4)}$. Em muitos pacientes, o diagnóstico inicial é inadequado, sendo estabelecido tardiamente no contexto diferencial de ataxia cerebelar aguda.

Descreve-se a seguir uma criança de dois anos e seis meses, encaminhada de outro serviço por quadro de encefalite pósviral ao Centro de Terapia Intensiva Pediátrica do Hospital Israelita Albert Einstein (HIAE), quando foi estabelecido o diagnóstico de SK. Durante a investigação e a exclusão paralela de neuroblastoma, a criança apresentou melhora progressiva e espetacular após a introdução de dexametasona.

O reconhecimento dessa doença é de suma importância, em vista da conhecida associação com neuroblastoma oculto, suas implicações prognósticas e devido a uma proporção considerável de pacientes que respondem clinicamente por meio de tratamento imunossupressor, como esteroides e imunoglobulina, entre outras terapias imunomodulatórias ${ }^{(5,6)}$.

\section{Descrição do caso}

Paciente de dois anos e seis meses, sexo feminino, branca, natural e procedente de Foz do Iguaçu (Paraná), previamente hígida, apresentou há 20 dias quadro de febre não medida e lesões em mãos, pés e boca. Procurou serviço médico que levantou a hipótese de síndrome mão-pé-boca, sendo orientadas medicações sintomáticas e observação em domicílio. Após cinco dias, a paciente foi internada com quadro de dificuldade para deambular, ataxia, nistagmo, estrabismo, irritabilidade e dificuldades articulatórias na fala. Realizou tomografia de crânio e coleta de líquido cefalorraquidiano que resultaram normais. Na ocasião, foi introduzido aciclovir, fenobarbital e ceftriaxona (este, suspenso após sete dias). Concomitantemente, apresentava coriza e tosse intermitente pouco produtiva, evoluindo sem febre. Houve melhora do nistagmo e do estrabismo, entretanto, não apresentou adequada recuperação motora e da fala, mantendo quadro de irritabilidade significativa associada. Por sugestão do hospital de origem, foi orientada a transferência para hospital mais especializado, com hipótese de encefalite pós-viral e quadro de cerebelite.

No serviço do HIAE, o exame clínico demonstrava a presença de opsomioclonias, irritabilidade e ataxia evidentes. A criança encontrava-se em regular estado geral, corada, hidratada, eupneica, acianótica, anictérica, afebril, consciente, irritada, agitada e chorosa à manipulação. Os parâmetros vitais iniciais demonstraram oximetria normal; frequência cardíaca de 150 batimentos por minuto (90 bpm ao sono); frequência respiratória de 20 incursões/minuto; peso de $12,2 \mathrm{~kg}$ (escore $Z=0,54$ desvios padrão); estatura: $92 \mathrm{~cm}$ (escore $Z=-0,56$ desvios padrão); pressão arterial de $107 \times 74 \mathrm{mmHg}$ (percentil 90-95).

Além de opsomioclonias, o exame neurológico evidenciou tremores aos movimentos dos membros superiores. A pesquisa dos demais nervos cranianos foi normal, os reflexos profundos estavam presentes e simétricos, com presença de reflexo cutâneo-plantar em flexão, além de não haver déficits motores. A escala de coma de Glasgow era 12 (abertura ocular $=4$; resposta verbal $=3$; resposta motora $=5$ ). Exames de hemograma, bioquímica, gasometria e eletrólitos foram normais. Realizou-se ressonância magnética de cabeça e tomografias de tórax e abdome para afastar a possibilidade de neuroblastoma. Esses exames de imagem evidenciaram áreas laminares de atelectasias pulmonares esparsas, predominantemente em pulmão esquerdo, presença de bile espessa (provavelmente relacionada ao uso prévio de ceftriaxona), não se detectando sinais de neuroblastoma.

$\mathrm{Na}$ apresentação clínica inicial, foi estabelecida a hipótese diagnóstica de SK, sendo suspensos o aciclovir e o fenobarbital. Introduzidos: dexametasona $1 \mathrm{mg}$ a cada seis horas por via parenteral $(0,33 \mathrm{mg} / \mathrm{kg} / \mathrm{dia})$, dieta hipossódica, ranitidina, inalação com soro fisiológico e fisioterapia respiratória. Realizado eletroencefalograma em vigília por 40 minutos, que demonstrou atividade de base normal para a idade, ritmo de predomínio posterior a $7-8 \mathrm{~Hz}, 20-60 \mu v$, presença de opsomioclonias sem mudanças de ritmos eletrográficos e ausência de paroxismos anormais. Sorologia para coxsackie B (ELISA) não indicou infecção recente. Posteriormente, foi solicitada 
a pesquisa de ácido vanilmandélico na urina, que resultou normal (amostra isolada: $<1 \mathrm{mg} / \mathrm{L} ; 0 \mathrm{mg} / \mathrm{g}$ de creatinina).

A criança evoluiu com níveis pressóricos normais, ausência de comprometimento cardíaco e melhora clínica do quadro de irritabilidade e da opsoclonia, sendo transferida para enfermaria, onde permaneceu mais calma e coordenada. Recebeu alta hospitalar após sete dias de internação com dexametasona e ranitidina via oral. Durante acompanhamento ambulatorial, a criança evoluiu com melhora progressiva e desaparecimento do quadro de ataxia e das opsomioclonias, sendo suspensas as medicações após seis meses de acompanhamento devido à acentuada síndrome cushingoide.

\section{Discussão}

A SK ou síndrome opsoclonia-mioclonia-ataxia é uma doença rara, caracterizada por opsoclonia (movimentos oculares conjugados rápidos, multidirecionais e anárquicos), ataxia cerebelar axial e apendicular e, algumas vezes, componente mioclônico com abalos musculares erráticos, podendo afetar o tronco, a face e as extremidades ${ }^{(1,2)}$. Em geral, existe irritabilidade significativa, cursando como pródromo mais frequente em algumas séries relatadas na literatura ${ }^{(2)}$. Outras manifestações frequentemente registradas são distúrbios do sono, alterações da personalidade, irritabilidade, disfasia, disartria, sialorreia, tremor intencional, mutismo, letargia, distúrbios deglutição, entre outros sintomas variáveis. Tais achados podem ser avaliados por escalas de graduação específicas para essa enfermidade ${ }^{(7)}$. Pode-se evidenciar também a regressão do desenvolvimento neuromotor em grande número de casos durante o seguimento, resultando em sequelas neurológicas variáveis ${ }^{(5,8,9)}$. Essa síndrome é mais frequentemente registrada em adultos do que em crianças $^{(9)}$. Em pediatria, a faixa etária acometida varia, em geral, entre seis e 36 meses de idade (média de 19 meses); entretanto, várias vezes o diagnóstico é realizado tardiamente ${ }^{(3)}$. Muitos casos são confundidos com encefalite ou cerebelite pós-viral, como no presente caso, fazendo parte do diagnóstico diferencial de ataxia cerebelar ${ }^{(2)}$.

Devido à raridade da SK, à possibilidade de natureza fugaz do quadro de opsoclonia e à ausência de um teste marcador diagnóstico, a epidemiologia e a história natural dessa enfermidade denotam um cenário de dificuldade de caracterização diagnóstica e epidemiológica. Na Inglaterra, por meio de questionário prospectivo envolvendo 20 centros de neurologia pediátrica entre 2003 e 2005, registraram-se
19 casos, obtendo-se uma incidência de 0,18 casos/ano/ milhão da população total ${ }^{(6)}$.

A etiologia é variada, incluindo causas idiopáticas, síndromes parainfecciosas (virais e bacterianas), metabólicas ou tóxicas (tálio, tolueno, organofosforados, lítio, cocaína etc.) e síndromes paraneoplásicas ${ }^{(5)}$. Em 40 a $80 \%$ dos casos, pode-se detectar um neuroblastoma oculto ${ }^{(2,6,9,10)}$, constituindo uma síndrome paraneoplásica. Raramente evidenciam-se em adultos outros tipos de neoplasias, como linfoma não Hodgkin, adenocarcinoma renal e melanoma, entre outros ${ }^{(5,11)}$. A SK também pode se associar ao pós-operatório de transplante de células tronco hematopoiéticas ${ }^{(12)}$.

O diagnóstico de neuroblastoma é detectado tardiamente, com uma mediana de três meses ${ }^{(2)}$. A localização preferencial do tumor é abdominal, torácica ou pélvica ${ }^{(2,9)}$. Na maioria dos casos, o diagnóstico de SK leva à revelação do neuroblastoma, entretanto, raramente os sinais clínicos de SK podem aparecer secundariamente ao diagnóstico do tumor ${ }^{(9)}$. Na maioria das vezes, os tumores em pacientes com SK são pequenos, apresentam características biológicas de baixa malignidade, demonstrando histologia favorável, estágios I-II, além de baixos níveis ou ausência de associação com MYC protooncogene e metástases, em comparação a tumores similares em crianças sem sintomatologia associada à SK ${ }^{(6,10,13,14)}$. Dessa forma, especula-se que o neuroblastoma poderia regredir espontaneamente antes da detecção da opsomioclonia.

Embora seja possível evidenciar um agente viral associado em alguns casos, não se considera a SK uma doença infecciosa ou de base genética ou hereditária. A patogênese dessa síndrome ainda está indefinida; entretanto, na maioria dos casos, observa-se um distúrbio neurológico autoimune. Mecanismos envolvendo distúrbios da imunidade celular e humoral parecem estar implicados na opsoclonia ${ }^{(5)}$. Em casos de síndrome paraneoplásica e SK, tanto em adultos como em crianças, é descrita a presença de autoanticorpos, incluindo anti-Ri, anti-Yo (PCA-1), anti-Hu (ANNA-1), anti-Ma1, anti-Ma2, antineurofilamentos, antineurolicinas e antigliadinas, entre outros ${ }^{(5)}$. Alguns desses autoanticorpos podem reconhecer e se fixar de forma específica a antígenos neuronais de superfície e intracelulares de linhagens de células de neuroblastomas, inibindo sua proliferação e induzindo à apoptose ${ }^{(15,16)}$. Em casos de SK e neuroblastoma, inúmeros autoanticorpos séricos contra neurônios e células cerebelares de Purkinje também podem ser detectados ${ }^{(16)}$. Evidenciam-se também linfócitos T e B ativados no líquor de crianças com essa enfermidade ${ }^{(17)}$. Análises imunoistoquímicas de amostras teciduais de neuroblastoma em 
crianças acometidas demonstram a presença de infiltrado linfoide intersticial e perivascular, similares a folículos linfóides secundários. A imunofenotipagem das células linfoides denota a existência de células dendríticas CD $21^{+}$, linfócitos $\mathrm{B} \mathrm{CD} 20^{+}$e linfócitos $\mathrm{T} \mathrm{CD} 3^{+}$. Essas evidências levantam a hipótese de um distúrbio de reação cruzada autoimune entre o neuroblastoma e o sistema nervoso central na SK e a participação de quimiocinas, entre outros distúrbios do sistema imunológico ${ }^{(16)}$.

Nos casos sem demonstração do tumor, frequentemente há relato de um pródromo viral, consistindo de sintomatologia de vias aéreas ou de origem gastrintestinal ${ }^{(2)}$. Ocasionalmente, a presença de um vírus neurotrópico pode ser isolado, por exemplo, coxsackie B, Epstein-Barr, parvovírus B19, enterovírus e vírus da encefalite de Saint Louis. A associação de opsoclonia e infecção por HIV também é relatada ${ }^{(18)}$. Infecções bacterianas podem estar presentes, como por Streptococcus pyogene e Mycoplasma pneumoniae, entre outros agentes ${ }^{(19,20)}$. Entretanto, mesmo a presença de um pródomo viral não afasta a necessidade de exclusão de neuroblastoma oculto ${ }^{(6,9)}$.

Em nosso meio, Kok, em uma série de 19 pacientes acompanhados por dez anos, observou duas formas de apresentações clínicas. Num primeiro grupo, os pacientes evoluíram de forma benigna, não recorrente e autolimitada, sendo possível detecção de algum agente infeccioso associado (enterovírus e varicela-zóster). Outro grupo foi constituído de pacientes com sintomatologia prolongada e recorrente, observandose sequelas neurológicas em longo prazo ${ }^{(19)}$. Nesse segundo grupo, houve associação com neuroblastoma em 3/14 casos. Dessa forma, torna-se obrigatória a investigação de neoplasia oculta nessa enfermidade. Foram observados relatos nacionais de SK, um deles sem associação e outro com neuroblastoma, evidenciado em tomografia abdominal ${ }^{(20,21)}$.

A maioria dos casos de SK inicia-se de forma insidiosa (dias a semanas de duração), com sintomatologia inicial inespecífica, como irritabilidade e mal-estar e, provavelmente, existem subtipos de apresentação e evolução, como já comentado previamente.

\section{Referências bibliográficas}

1. Kinsbourne M. Myoclonic encephalopathy of infants. J Neurol Neurosurg Psychiatry 1962;25:271-6.

2. Tate DE, Allison TJ, Pranzatelli MR, Verhulst SJ. Neuroepidemiologic trends in 105 US cases of pediatric opsoclonus-myoclonus syndrome. J Pediatr Oncol Nurs 2005;22:8-19.

3. Pranzatelli MR. The neurobiology of the opsoclonus-myoclonus syndrome. Clin Neuropharmacol 1992;15:186-228.
Embora sejam descritos casos de remissão espontânea e desenvolvimento normal ${ }^{(6)}$, muitos pacientes, devido à manutenção das manifestações neurológicas, recebem tratamento imunossupressor, além de ressecção e/ou tratamento quimioterápico, quando detectado o tumor ${ }^{(2,10,16)}$. Dentre os agentes quimioterápicos empregados, estão a ciclofosfami$\mathrm{da}$, a carboplatina, a doxorubicina e a VP16 $6^{(2,9)}$. As terapias com corticosteroide ou com hormônio adrenocorticotrófico (ACTH) são benéficas no controle de sintomas neurológicos nas formas recorrentes da doença e costumam ser a primeira linha de tratamento ${ }^{(9,22)}$. Em alguns casos, há necessidade de manutenção da terapia por vários meses ${ }^{(19)}$.

A gamaglobulina intravenosa, a azatioprina, a ciclosfosfami$\mathrm{da}$, as sessões de plasmaferese e, recentemente, o rituximabe são alternativas terapêuticas para casos refratários ${ }^{(2,9,23,24)}$. Em nosso meio, essas alternativas terapêuticas ainda são limitadas.

O prognóstico é variável. Entretanto, em crianças, o curso da SK é caracterizado, em geral, por recidivas múltiplas, requerendo tratamento prolongado, com sequelas de desenvolvimento neurológico significativas. Somente uma minoria de crianças apresenta um curso monofásico e um prognóstico mais benigno ${ }^{(8)}$. Na evolução, são relativamente comuns distúrbios de comportamento, cognitivos, de linguagem e aprendizagem, alterações do sono e problemas de ordem psiquiátrica ${ }^{(2)}$.

A paciente do caso aqui relatado apresentou quadro neurológico inicial dramático de opsomioclonia e ataxia, com sintomas agudos após provável virose. Até o momento, não foi detectada neoplasia associada e a introdução de corticoterapia demonstrou evolução surpreendente, com resolução da sintomatologia e ausência de sequelas neurológicas. Apesar de se tratar de doença rara, o diagnóstico de SK deve ser reconhecido pelos pediatras e intensivistas no diagnóstico diferencial de ataxia cerebelar aguda. É fundamental a exclusão de neuroblastoma oculto e o tratamento específico precoce, embora com resultados variáveis, pode, em alguns casos, resultar em evolução satisfatória, como observado na paciente aqui relatada.

4. Farrelly C, Daneman A, Chan HS, Martin DJ. Occult neuroblastoma presenting with opsomyoclonus: utility of computed tomography. AJR 1984;142:807-10. 5. Wong A. An update on opsoclonus. Curr Opin Neurol 2007;20:25-31.

6. Pang KK, de Sousa C, Lang B, Pike MG. A prospective study of the presentation and management of dancing eye syndrome/opsoclonusmyoclonus syndrome in the United Kingdom. Eur J Paediatr Neurol 2010;14:156-61. 
7. Matthay KK, Blaes F, Hero B, Plantaz D, De Alarcon P, Mitchell WG et al. Opsoclonus myoclonus syndrome in neuroblastoma a report from a workshop on the dancing eyes syndrome at the advances in neuroblastoma meeting in Genoa, Italy, 2004. Cancer Lett 2005;228:275-82.

8. Mitchell WG, Brumm VL, Azen CG, Patterson KE, Aller SK, Rodriguez J. Longitudinal neurodevelopmental evaluation of children with opsoclonusataxia. Pediatrics 2005;116:901-7.

9. Krug P, Schleiermacher G, Michon J, Valteau-Couanet D, Brisse H, Peuchmaur $\mathrm{M}$ et al. Opsoclonus-myoclonus in children associated or not with neuroblastoma. Eur J Paediatr Neurol 2010;14:400-9.

10. Rothenberg AB, Berdon WE, D'Angio GJ, Yamashiro DJ, Cowles RA. The association between neuroblastoma and opsoclonus-myoclonus syndrome: a historical review. Pediatr Radiol 2009;39:723-6.

11. Pohl KR, Pritchard J, Wilson J. Neurological sequelae of the dancing eye syndrome. Eur J Pediatr 1996;155:237-44.

12. Bishton MJ, Das Gupta E, Byrne JL, Russell NH. Opsoclonus myoclonus following allogeneic haematopoietic stem cell transplantation. Bone Marrow Transplant 2005;36:923.

13. Altman AJ, Baehner RL. Favorable prognosis for survival in children with coincident opso-myoclonus and neuroblastoma. Cancer 1976;37:846-52.

14. Cohn SL, Salwen H, Herst CV, Maurer HS, Nieder ML, Morgan ER et al. Single copies of the $\mathrm{N}$-myc oncogene in neuroblastomas from children presenting with the syndrome of opsoclonus-myoclonus. Cancer 1988;62:723-6.

15. Korfei M, Fühlhuber V, Schmidt-Wöll T, Kaps M, Preissner KT, Blaes F. Functional characterisation of autoantibodies from patients with pediatric opsoclonus-myoclonus-syndrome. J Neuroimmunol 2005;170:150-7.

16. Raffaghello L, Conte M, De Grandis E, Pistoia V. Immunological mechanisms in opsoclonus-myoclonus associated neuroblastoma. Eur J Paediatr Neurol 2009;13:219-23.

17. Pranzatelli MR, Travelstead AL, Tate ED, Allison TJ, Moticka EJ, Franz DN et al. B- and T-cell markers in opsoclonus-myoclonus syndrome: immunophenotyping of CSF lymphocytes. Neurology 2004;62:1526-32.

18. Van Toorn R, Rabie H, Warwick JM. Opsoclonus-myoclonus in an HIV-infected child on antiretroviral therapy-possible immune reconstitution inflammatory syndrome. Eur J Paediatr Neurol 2005;9:423-6.

19. Kok F. Ataxias agudas. In: Diament A, Cypel S, Reed UC, editors. Neurologia infantil. $5^{\text {th }}$ ed. São Paulo: Atheneu; 2010. p. 1677-9.

20. Alves R, Lovatelli R. Síndrome de Opsoclonus-mioclonus-ataxia: relato de caso. Pediatria (São Paulo) 2007;29:150-3.

21. Oliver KA, Bruc I, Santos LH, Antoniuk AS, Bodanese MJ. Opsoclonusmioclonus: uma manifestação clínica aguda de neuroblastoma. Pediatria (São Paulo) 2010;32:67-70

22. Rosemberg S. Movimentos involuntários anormais. In: Rosemberg S, editor. Neuropediatria. $2^{\text {nd }}$ ed. São Paulo: Sarvier; 2010. p. 47-63.

23. Pranzatelli MR, Tate ED, Travelstead AL, Longee D. Immunologic and clinical responses to rituximab in a child with opsoclonus-myoclonus syndrome. Pediatrics 2005;115;e115-9.

24. Leen WG, Weemaes CM, Verbeek MM, Willemsen MA, Rotteveel JJ. Rituximab and intravenous immunoglobulins for relapsing postinfecitous opsoclonusmyoclonus syndrome. Pediatr Neurol 2008;213-7. 